\title{
Chest tube management in patients undergoing lobectomy
}

\author{
Yasushi Shintani, Soichiro Funaki, Naoko Ose, Takashi Kanou, Ryu Kanzaki, Masato Minami, \\ Meinoshin Okumura
}

Department of General Thoracic Surgery, Osaka University Graduate School of Medicine, Osaka, Japan

Correspondence to: Yasushi Shintani, MD, PhD. Department of General Thoracic Surgery, Osaka University Graduate School of Medicine, 2-2-L5, Yamadaoka, Suita, Osaka 565-0871, Japan. Email: yshintani@thoracic.med.osaka-u.ac.jp.

Comment on: Gao S, Zhang Z, Aragón J, et al. The Society for Translational Medicine: clinical practice guidelines for the postoperative management of chest tube for patients undergoing lobectomy. J Thorac Dis 2017;9:3255-64.

Submitted Nov 05, 2018. Accepted for publication Nov 06, 2018.

doi: $10.21037 /$ jtd.2018.11.47

View this article at: http://dx.doi.org/10.21037/jtd.2018.11.47

\section{Introduction}

Full expansion of remaining lung tissue is the most important issue following a pulmonary lobectomy, for which chest tubes are used for proper drainage of both air and fluid, with the outflow considered to reflect the condition of the thoracic cavity after surgery $(1,2)$. Postoperative management of chest tubes is largely based on protocols developed by individual institutions or surgeons. In a collaborative study presented in 2011, the European Society of Thoracic Surgeons (ESTS), American Association for Thoracic Surgery (AATS), Society of Thoracic Surgeons (STS), and General Thoracic Surgery Club (GTSC) proposed standardized definitions and nomenclature for chest tube management (3). In addition, the Society for Translational Medicine and The Chinese Society for Thoracic and Cardiovascular Surgery have also produced recommendations for postoperative management of chest tubes in patients who underwent a pulmonary lobectomy according to a systematic review of literature (2). These proposals have been helpful for decision making regarding clinical management of patients who undergo a pulmonary resection. In addition, in the present report we propose additional recommendations for chest tube drainage after a pulmonary lobectomy based on our experience as well as a review of relevant studies.

\section{Positioning of chest tubes}

Immediately following lung resection, air tends to collect in the retrosternal part of the chest wall (supine position) and fluid in the lower part (costodiaphragmatic sinus).
Thus, several current general thoracic surgery textbooks recommend placement of 2 chest tubes after a major pulmonary resection procedure, one in an anterior position to remove air, and the other in the posterior and basilar region to drain fluid (4). On the other hand, recent studies have found that use of a single chest tube is effective for lung expansion and reducing postoperative pain, resulting in adequate respiratory physiotherapy and decreasing the risk of possible respiratory complications $(5,6)$. As a result, the general protocol used by many thoracic surgeons includes placement of only one chest tube after a pulmonary lobectomy, as the attending surgeon has direct knowledge of which part of the thoracic cavity requires drainage after that procedure. At our institution, we routinely place a single chest tube after a pulmonary lobectomy according to the following protocol. Following an upper or middle lobectomy, we place the chest tube in the upper area of the thoracic cavity, while we place that in the lower area for both air and fluid drainage following a lower lobectomy. In patients with massive air leakage, pleural effusion, or hemorrhage, two chest tubes are routinely used.

\section{Types of chest tubes}

Conventional chest tubes are made of plastic or silastic materials, and are straight with an end hole and series of side holes. Since the length of the tube to the side holes is the effective drainage length, surgeons often fashion additional side holes manually according to the adequate drainage point. Size varies according to the manufacturer and surgeons determine which is best depending on the 

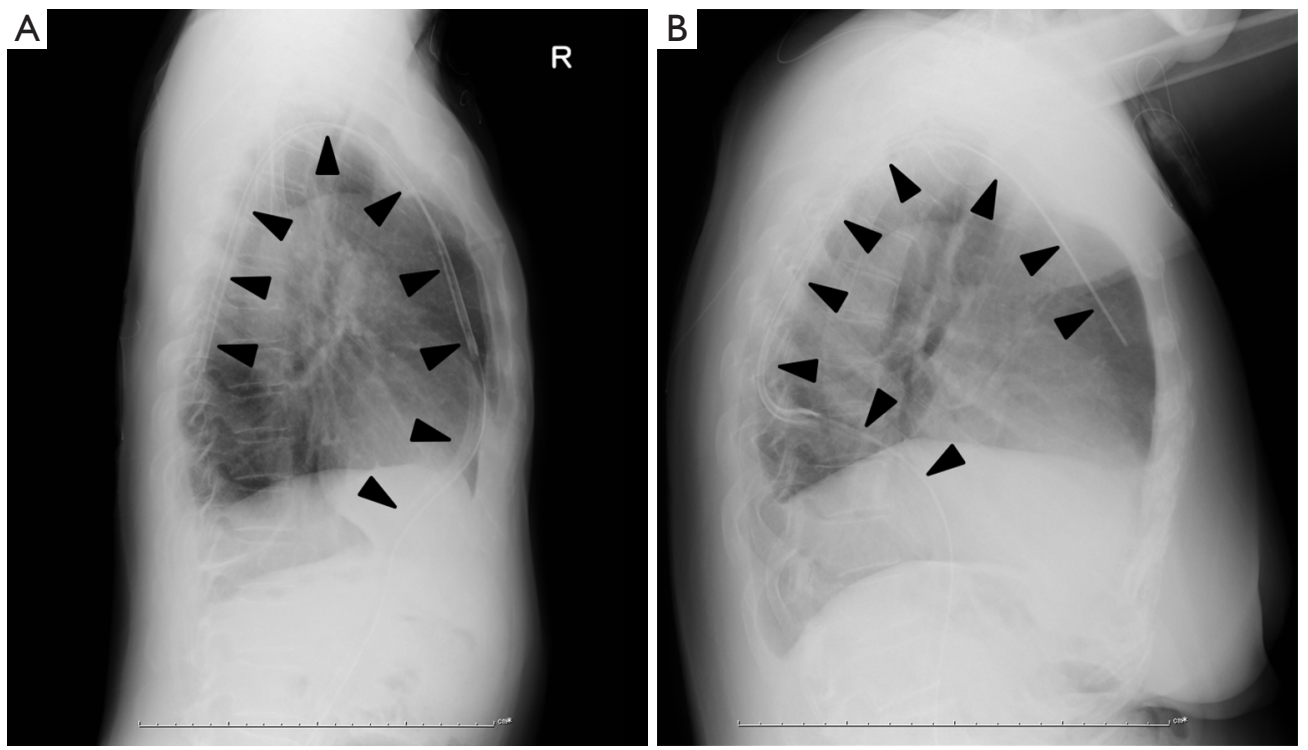

Figure 1 Representative chest roentgenogram images. (A) Blake drainage tube inserted from anterior apical portion of lung descending into dorsal region after right upper lobectomy; (B) Blake drainage tube inserted from posterior portion of lung ascending into visceral region after lower lobectomy.

situation of the individual case after a pulmonary resection. For example, following dissection of a severe adhesion in the lung, a thick drain is preferred for adequate drainage of effusion and hemorrhage.

Blake drains are a round type of flexible silicone tube that have been reported to be as effective as traditional chest tubes for thoracic drainage $(1,7)$. They are equipped with grooves that run along the tube length and the fluted design is considered suitable to promote fluid drainage (8). Since a Blake drain exerts constant suction over the entire length of the fluted portion, both air and fluid can be evacuated. Air is drained mostly using the proximal site of the fluted portion of the drain, thus we prefer to insert the drain at the $7^{\text {th }}$ intercostal middle axillary line descending into the dorsal region via the anterior apical portion of the lung after an upper or middle lobectomy, or ascending into the visceral region via the posterior portion of the lung following a lower lobectomy (Figure 1).

\section{Air leakage}

One of the most common causes of complications after a pulmonary resection is prolonged air leakage $(9,10)$. Management of a pulmonary air leak is an important clinical issue for thoracic surgeons (11), as it often leads to increased complications, including longer drainage time, infection, and empyema when prolonged, resulting in an extended hospitalization (12).

The effectiveness of a test tube with or without suction is controversial in regard to reducing the duration of air leakage, and the choice is generally left up to the attending surgeon (13). A tube with suction provides external suction, while no suction indicates a water seal. Several studies have reported that external suction offers no advantage for patients who have undergone a lobectomy (14), thus it is common practice to first directly apply external suction to chest tubes following a pulmonary resection to enhance pleural apposition and then switch to a water seal as soon as possible.

A digital pleural drainage system can be used to maintain a preset intrathoracic pressure, termed regulated pressure, and is associated with significant reductions in air leak duration, chest tube placement duration, and postoperative hospitalization $(14,15)$. On the other hand, using findings from a prospective randomized trial conducted to evaluate the effectiveness of a digital thoracic drainage system, Takamochi et al. concluded that use of a such a system did not shorten the duration of chest tube placement as compared to a traditional thoracic drainage system following anatomic lung resection procedures (16). On the other hand, a digital pleural drainage system can provide information regarding the amount of air being leaked, as 
well as monitoring of peak air leakage and leakage patterns, which are useful for predicting the period of treatment and related decision making after a pulmonary resection $(17,18)$.

\section{Chest tube removal}

The most common complication of chest tube removal is lung collapse and whether to clamp a chest tube prior to removal remains a controversial issue. Although several studies have reported no benefit of chest tube clamping prior to removal (19), many physicians prefer to clamp the chest tube for detecting the presence of a small amount of air leakage. In studies that utilized a digital thoracic drainage system, several threshold conditions for chest tube removal have been proposed, such as air leak $<20 \mathrm{~mL} /$ minute (9), or $<40 \mathrm{~mL} /$ minute for 6 hours (20) or $<30 \mathrm{~mL} /$ minute for $>8$ hours (21). These criteria might depend on physician experience. Nevertheless, since a digital thoracic drainage system shows air leakage trend, a clamp test is considered to be unnecessary.

In patients without air leakage or bloody, chylous, or purulent pleural effusion, chest tube removal depends on drainage volume over a period of 24 hours, though the threshold for a safe volume indicating chest tube removal after a pulmonary lobectomy remains controversial. Many thoracic surgeons use the threshold of $100-250 \mathrm{~mL} /$ day for indicating such removal, though several authors have reported that a chest tube can be removed safely when the daily pleural fluid amount is up to $450 \mathrm{~mL}$, and without hematic or chylous contents (2). Nakanishi et al. also evaluated appropriate drainage volume for indicating chest tube removal, and concluded that early removal of a chest tube on the day after a thoracoscopic lobectomy regardless of drainage volume appears to be safe in patients without air leakage or bloody, chylous, or purulent pleural effusion (22). In other studies, Ueda et al. (23) and Murakami et al. (24) investigated omission of postoperative chest tube drainage in selected patients undergoing lung resection. They carefully assessed air leaks until removal of the tracheal tube and then removed the chest tube in the operating room if none were detected. Each study concluded that omission of chest tube drainage is feasible in the majority of patients undergoing thoracoscopic major lung resection without increasing the risk of adverse events.

\section{Conclusions}

Introduction of new chest tube types and drainage systems has made innovative contributions to thoracic surgery. It is important for surgeons to remain abreast of current knowledge regarding postoperative chest tube management in their patients following a pulmonary resection procedure.

\section{Acknowledgements}

None.

\section{Footnote}

Conflicts of Interest: The authors have no conflicts of interest to declare.

\section{References}

1. Nakamura H, Taniguchi Y, Miwa K, et al. The use of Blake drains following general thoracic surgery: is it an acceptable option? Interact Cardiovasc Thorac Surg 2009;8:58-61.

2. Gao S, Zhang Z, Aragon J, et al. The Society for Translational Medicine: clinical practice guidelines for the postoperative management of chest tube for patients undergoing lobectomy. J Thorac Dis 2017;9:3255-64.

3. Brunelli A, Beretta E, Cassivi SD, et al. Consensus definitions to promote an evidence-based approach to management of the pleural space. A collaborative proposal by ESTS, AATS, STS, and GTSC. Eur J Cardiothorac Surg 2011;40:291-7.

4. Filosso PL, Sandri A, Guerrera F, et al. Management of Chest Drains After Thoracic Resections. Thorac Surg Clin 2017;27:7-11.

5. Tanaka M, Sagawa M, Usuda K, et al. Postoperative drainage with one chest tube is appropriate for pulmonary lobectomy: a randomized trial. Tohoku J Exp Med 2014;232:55-61.

6. Zhou D, Deng XF, Liu QX, et al. Single chest tube drainage is superior to double chest tube drainage after lobectomy: a meta-analysis. J Cardiothorac Surg 2016;11:88.

7. Gordon BM, Hasaniya NW, Newcombe JB, et al. Blake drains: a novel method of chest drainage after extracardiac fontan operation with autologous pericardium. Ann Thorac Surg 2012;94:1289-94.

8. Ishikura H, Kimura S. The use of flexible silastic drains after chest surgery: Novel thoracic drainage. Ann Thorac Surg 2006;81:331-3.

9. Filosso PL, Nigra VA, Lanza G, et al. Digital versus 
traditional air leak evaluation after elective pulmonary resection: a prospective and comparative monoinstitutional study. J Thorac Dis 2015;7:1719-24.

10. Burt BM, Shrager JB. Prevention and management of postoperative air leaks. Ann Cardiothorac Surg 2014;3:216-8.

11. Brunelli A, Cassivi SD, Halgren L. Risk factors for prolonged air leak after pulmonary resection. Thorac Surg Clin 2010;20:359-64.

12. Anegg U, Rychlik R, Smolle-Jüttner F. Do the benefits of shorter hospital stay associated with the use of fleecebound sealing outweigh the cost of the materials?. Interact Cardiovasc Thorac Surg 2008;7:292-6; discussion 226.

13. Deng B, Tan QY, Zhao YP, et al. Suction or non-suction to the underwater seal drains following pulmonary operation: meta-analysis of randomised controlled trials. Eur J Cardiothorac Surg 2010;38:210-5.

14. Bertholet JW, Joosten JJ, Keemers-Gels ME, et al. Chest tube management following pulmonary lobectomy: change of protocol results in fewer air leaks. Interact Cardiovasc Thorac Surg 2011;12:28-31.

15. Cerfolio RJ, Bryant AS. The benefits of continuous and digital air leak assessment after elective pulmonary resection: a prospective study. Ann Thorac Surg 2008;86:396-401.

16. Takamochi K, Nojiri S, Oh S, et al. Comparison of digital and traditional thoracic drainage systems for postoperative chest tube management after pulmonary resection: A prospective randomized trial. J Thorac Cardiovasc Surg

Cite this article as: Shintani Y, Funaki S, Ose N, Kanou T, Kanzaki R, Minami M, Okumura M. Chest tube management in patients undergoing lobectomy. J Thorac Dis 2018;10(12):64326435. doi: $10.21037 / j t d .2018 .11 .47$
2018;155:1834-40.

17. Takamochi K, Imashimizu K, Fukui M, et al. Utility of Objective Chest Tube Management After Pulmonary Resection Using a Digital Drainage System. Ann Thorac Surg 2017;104:275-83.

18. Shintani Y, Funaki S, Ose N, et al. Air leak pattern shown by digital chest drainage system predict prolonged air leakage after pulmonary resection for patients with lung cancer. J Thorac Dis 2018;10:3714-21.

19. Laws D, Neville E, Duffy J, et al. BTS guidelines for the insertion of a chest drain. Thorax 2003;58 Suppl 2:ii53-9.

20. George RS, Papagiannopoulos K. Advances in chest drain management in thoracic disease. J Thorac Dis 2016;8:S55-64.

21. Pompili C, Detterbeck F, Papagiannopoulos K, et al. Multicenter international randomized comparison of objective and subjective outcomes between electronic and traditional chest drainage systems. Ann Thorac Surg 2014;98:490-6; discussion 496-7.

22. Nakanishi R, Fujino Y, Kato M, et al. Early chest tube removal after thoracoscopic lobectomy with the aid of an additional thin tube: a prospective multi-institutional study. Gen Thorac Cardiovasc Surg 2018;66:723-30.

23. Ueda K, Hayashi M, Tanaka T, et al. Omitting chest tube drainage after thoracoscopic major lung resection. Eur J Cardiothorac Surg 2013;44:225-9; discussion 229.

24. Murakami J, Ueda K, Tanaka T, et al. The Validation of a No-Drain Policy After Thoracoscopic Major Lung Resection. Ann Thorac Surg 2017;104:1005-11. 